\title{
LETTERS
}

\section{Doctor, physician, professor, teacher}

In their letter responding to a CMAJ news article, ${ }^{1}$ Drs. Asfour and Winter bring up the interesting question of "who is a doctor?"2

Descriptively, the answer depends on jurisdiction. In several European nations, such as Germany and Poland, a doctor is a holder of an academic doctorate. Graduates of undergraduate medical education who complete an internship and pass national examinations are called "physicians" - and not "doctors." Many do complete a doctorate in medicine or medical sciences, and for this reason - alongside the historical association of medical practitioners with these degrees recently graduated physicians may be addressed as "doctor"; however, they are not allowed to call themselves as such by legislation.

But keeping the above in mind, the answer to the closely related question "who is a physician?" also depends on jurisdiction. Given the historical difference between practitioners of medicine, there are jurisdictions where a "physician" is a specialist in medicine, or at least one who is a member of a college of physicians. This is meant to make clear the distinction between specialists and surgeons, psychiatrists, general practitioners or junior medical practitioners working in any field, including junior medical trainees who have not gained admission to a college of physicians. All of these are known simply as "doctors." In other systems, they are all physicians, as all medical practitioners, including surgeons, study medicine.

Interestingly, even the question of "who is a professor?" depends on context. In the United Kingdom, Poland and Germany, the holder of an academic chair is a professor. In the UK, they may also be pro- fessor emeritus. In Germany and Poland, they are called "professor" only if they have been awarded the academic degree of professor (and holders of such are always professors, regardless of academic position). In Canada and the United States, there are full professors who do not hold either the aforementioned degree or even an academic doctorate, but rather professional degrees in medicine, so that presenting research findings in Berlin or Szczecin may result in the humorous scenario that one may be, at the same time, a professor but not a doctor.

Australia has attempted to provide some clarity to the public in distinguishing between academic doctors and medical practitioners by restricting this latter term in legislature. But ensuring clarity for a public exposed to globalized popular culture may be difficult, as well as among an educated class of academics when exposed to different contexts through conferences and literature. Finding such clarity for English-speaking audiences might require agreements between various parties in, at the very least, Canada, the US, the UK, the European Union and potentially also India. All of these might be accustomed to their own traditions.

In normative terms, answering the question of "who should be called a doctor," even limiting ourselves to the premise that current usage should reflect historical usage of the Latin word, may depend on knowing more beyond this context. Going back to the so-called medieval universities, was the term "doctor" used to refer to serving faculty with doctoral degrees? If so, it could be argued that neither the medical practitioner in the UK who has only an undergraduate degree in medicine, nor the Doctor of Philosophy degree-holder working in industry without an academic appointment, should be called doctors. We might wish to look even further back in time to when the Latin "docere" became "doctor." If at this time a "doctor" is one whose occupation is the conduction of formal instruction to students, then the faculty of an elementary school might be called "doctors," but not so the academic doctorate-holder working in industry or clinical medicine. However, if the term "doctor" at this time reflects the status of a learned person who "teaches" - whether undergraduates by giving lectures, graduates by supervising study, the academic world and the public at large by publishing research, or their patients by providing medical advice - both might be "doctors." Maybe, just maybe, we might decide that despite historical usage, this is the one we wish to adopt now. There may be an argument that a pharmacist holding a PharmD also educates, whether by providing information to patients in the community pharmacy, assisting medical practitioners to select medications in the hospital, or publishing in the literature. These approaches might be different in the same way that they are different for an academic, depending on whether they address undergraduate audiences, peers or the public. However, all cases require making use of achieved academic learning. Whether this learning is remotely equitable between the PharmD and PhD holder is not for myself, a very junior medical practitioner, to say.

\section{Konrad Marski BA MD}

Junior medical practitioner, Graduate of the Medical University of Łódź, Łódź, Poland

Cite as: CMAJ 2018 November 12;190: E1339. doi: 10.1503/cmaj.70447

\section{Reference}

1. Collier R. Who is entitled to the title of "doctor"? CMAJ 2016;188:E305.

2. Asfour A-F, Winter J. Whom should we really call a "doctor"? [letter]. CMAJ 2018;190:E660.

Competing interests: None declared. 